\title{
Modification on Shahad.A.H Distance for Images processing
}

\author{
${ }^{\text {I}}$ SHAHAD ADIL TAHER, ${ }^{2}$ HIND RUSTUM MOHAMMED \\ ${ }^{1}$ Asst.lecturer, Iraq, shahada.alkhafaji @uokufa.edu.iq \\ ${ }^{2}$ prof. , Iraq, hindrustum.shaaban@uokufa.edu.iq
}

\begin{abstract}
:
In our paper we presented a suggested method for encoding images based mainly on Shahad.A.H distance, and will name it modification of on Shahad.A.H (M Sh .A.H) and Compare with Euclid distance and distance Shahad.A.H The rate of error ratio between the original images and the methods presented was calculated and indicated that the modified method was the best in terms of performance evaluation criteria PSNR,SSIM,COR, NPCR, RMSE , our aim of this paper to develop Shahad.A.H To effectively hide image features ,Data base that independent in this paper (50 images) of different formats( .png,.bmp, .tif , .gif, jpg,), and multi types(color, gray) images and In paper MATLAB $® 2014$ software is used in the computer programming .
\end{abstract}

Keywords : Image processing, Image encryption, Distance Measure, Shahad.A.H Distance.

\section{INTRODUCTION}

The data can be protected either text, image and video by encrypting and transmitting information using mathematical equations and modern software technologies[1].

Encryption Image is one of the important tool for carrying information by applying encryption process information is encoded by the authorized persons schemes have been increasingly studied to meet demand for real time method secure image transmission over private or public networks [2].

There are so many different features used to measure image encryption range by find and analyzing Mathematical parameters are very important. The following values must be consider are key space, histogram analysis, correlation coefficient analysis, number of pixels change rate, PSNR, and MSE[3]. The paper is divided into 7 sections after the introduction comes the second section is the most prominent distances used and the third section represents the proposed method, Section 4 outlines a set of important definitions and terms that will be used in the paper
Section 5 Experiments and Results, While a description of the performance criteria in Section 6 , The 7th and final section means the conclusions.

\section{MATERIALS AND METHODS}

In this section we discuss the definitions of distance measures :

\section{Distance in matric space :}

Suppose we have the set called $\mathrm{Y}$, a function $1: \mathrm{Y} \times \mathrm{Y} \rightarrow \mathrm{R}$ is called distance on $\mathrm{Y}$ if $\forall \mathrm{s}, \mathrm{a} \in \mathrm{Y}$, then :

$1(\mathrm{~s}, \mathrm{a}) \geq 0$ (non-negativity)

$1(\mathrm{~s}, \mathrm{a})=1(\mathrm{a}, \mathrm{s})$ (symmetry)

$1(\mathrm{~s}, \mathrm{~s})=0 \quad$ (reflexivity) [4]

A most common distance measures:

Euclidean distance [5]:

Euclidean distance is defined by

$$
D_{\text {Fusideun }}\left(x_{i}, x_{j}\right)=\sqrt{\sum_{k-1}^{d}\left(x_{i k}-x_{j k}\right)^{2}}
$$

Shahad .A . H DISTANCE [6 ]

Let $\mathbf{X}, \mathbf{Y}$ are two points in two dimensional Euclidean spaces, the coordinates $\left(\mathbf{x}_{\mathbf{1}}, \mathbf{x}_{\mathbf{2}}\right)$ with $\mathbf{X}$, the coordinates $\left(\mathbf{y}_{1}, \mathbf{y}_{2}\right)$ with $\mathbf{Y}$.

The distance between $\mathrm{X}, \mathrm{Y}$ is defined by :

$$
\mathrm{D}(\mathrm{X}, \mathrm{Y})=\left|1-\tan \sqrt{\left(\mathrm{x}_{1}-\mathrm{y}_{1}\right)^{2}+\left(\mathrm{x}_{2}-\mathrm{y}_{2}\right)^{2}}\right|
$$

\section{PROPOSED METHOD}

For encryption image we used the inverse of Shahad.A . H distance method and multiplication with the mean value of all two pixels in image;

To apply the proposed method

we must

1- find the Eq 2 .

2-find the inverse of Eq 2. 
3-find the multiplication between $\mathrm{Eq} 2$ and mean value of the coordinates $\left(\mathbf{x}_{1}, \mathbf{x}_{2}\right),\left(\mathbf{y}_{1}, \mathbf{y}_{2}\right)$ of $\mathrm{X}$, Y respectively .

and we can calculated it by:

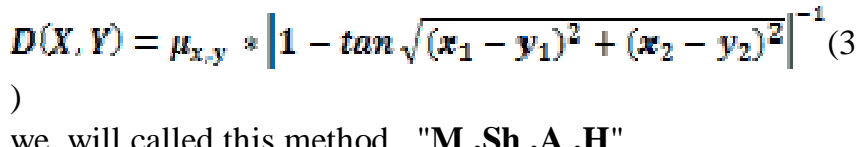

\section{SOME IMPORTANT DEFINITIONS}

\section{Peak Signal-To-Noise Ratio (PSNR) [7].}

$\mathrm{s}$ and $\mathrm{z}$ are two images (original image , test image) both of size $\mathrm{M} \times \mathrm{N}$, for $256^{*} 256$ images , Maximum intensity is $255(0$ to 255$)$.

$\operatorname{PSNR}(s, z)=10 \log _{10} \frac{255^{2}}{\operatorname{MSE}(s, z)}$

Where Mean square error (MSE )is defined by

MSE $=\frac{1}{m n} \sum_{i}^{m} \sum_{j}^{n}\left(s_{i j}-z_{i j}\right)^{2}$

Correlation Of Raw Scores [8],[14].

$\operatorname{com}=\mathrm{r}=\frac{\Sigma\left(\mathrm{d}_{\mathrm{i}}-\overline{\mathrm{d}}\right)\left(\mathrm{s}_{\mathrm{i}}-\overline{\mathrm{s}}\right)}{\left[\Sigma\left(\mathrm{d}_{\mathrm{i}}-\mathrm{d}\right)^{2} \Sigma\left(\mathrm{s}_{\mathrm{i}}-\bar{s}\right)^{2}\right]^{\frac{1}{2}}}$

where $\overline{d t}, \bar{s}$ are expected value of $d_{[}, s_{[}$respectively.

When $r=1,-1$ that means the Correlation coefficient is very strong in( positive and in negative) respectively .

If $\mathrm{r}=0$, then the Correlation coefficient is very week .

\section{structural similarity index[9].}

Structural similarity index defined by

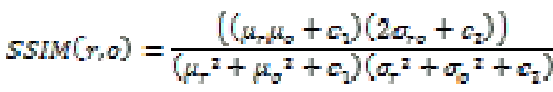

and it contain 3 comparison functions known for: luminance comparison, contrast comparison, and structure comparison between two signals $\mathbf{r}$ and $\mathbf{o}$.

Where

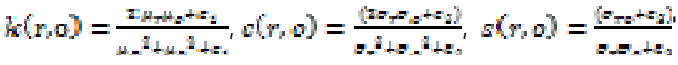

Where $\mu_{s}=\frac{1}{s} \sum_{i=2}^{s} r_{i, F} \mu_{s}=\frac{1}{s} \sum_{i=2}^{s} O_{i}$

Where $\boldsymbol{\mu}_{r}, \boldsymbol{\mu}_{\boldsymbol{v}}$ are the expected value of $\mathbf{r}$ and $\mathbf{o}$, and

$\sigma_{\mathrm{r}}=\left[\frac{1}{g-1} \sum_{i=1}^{s}\left(\eta_{i}-\mu_{\mathrm{r}}\right)^{2}\right]^{\frac{1}{2}}, \sigma_{\mathrm{z}}=\left[\frac{1}{g-1} \sum_{i=1}^{s}\left(\sigma_{i}-\mu_{z}\right)^{2}\right]^{\frac{1}{2}}$ $\boldsymbol{\sigma}_{a}, \boldsymbol{\sigma}_{b}$ are the standard deviations of $\mathbf{r}$ and $\mathbf{o}$, respectively, and $\boldsymbol{\sigma}_{\mathrm{ra}}$ is the correlation coefficient between $\mathbf{r}$ and $\mathbf{o}$. when the denominators approach to zero Then constants $c_{1}, c_{\mathbf{z}}$, and $c_{3}$ are used to stabilize the algorithm.

\section{Variance [10].}

If we have a set of values, then

The variance this set, which denote by $\sigma^{2}$, is defined as

$\sigma^{2}=\frac{\Sigma(z-\bar{z})^{2}}{m}$

where $\bar{t}$ is the expected value, $\mathbf{m}$ is the NO. of data values, and $\mathbf{t}$ stands for each data value in turn, An alternative, yet equivalent formula, which is often easier to use is

$\sigma^{2}=\frac{\Sigma t^{2}}{m}-\bar{t}^{2}$

Root Mean Square Error [11 ]:

$$
\text { RMSE }=\sqrt{\frac{\sum_{i=1}^{K}(z-s)^{2}}{\kappa}}
$$

Where $\mathrm{Z}$ is the reference value and $\mathrm{S}$ is the approximation value.

Histogram [12] .

$$
\mathrm{h}\left(\mathrm{q}_{\mathrm{c}}\right)=\mathrm{n}_{\mathrm{c}} \quad \quad=0,1, \ldots,-1
$$

$q_{\mathrm{c}}:$ the c-th gray level.

$n_{\varepsilon}:$ No. of times that the gray level $w_{c}$

\section{Histogram Equalization [13].}

$Y_{C}=G\left(r_{C}\right)=\sum_{l=0}^{C} \frac{m_{l}}{M}=\sum_{j=0}^{C} \boldsymbol{R}_{r}\left(r_{l}\right)$.

$0 \leq r_{\mathrm{c}} \leq 1, C=0,1,2, \ldots \ldots, l-1$

$\mathrm{m}_{\mathrm{j}}$ : the No. of times the $\mathrm{j}^{\text {th }}$ gray level.

$\mathrm{L}$ : NO. of gray levels.

$R_{r}\left(\eta_{j}\right)$ : the probability of the $\mathrm{j}^{\text {th }}$ gray level .

M: the total No. of pixels.

\section{EXPERIMENTAL and RESULTS}

We will analyze in this section the effect of some of the prescriptions on the components of the image as follows: 
Direct effect methods on original images .

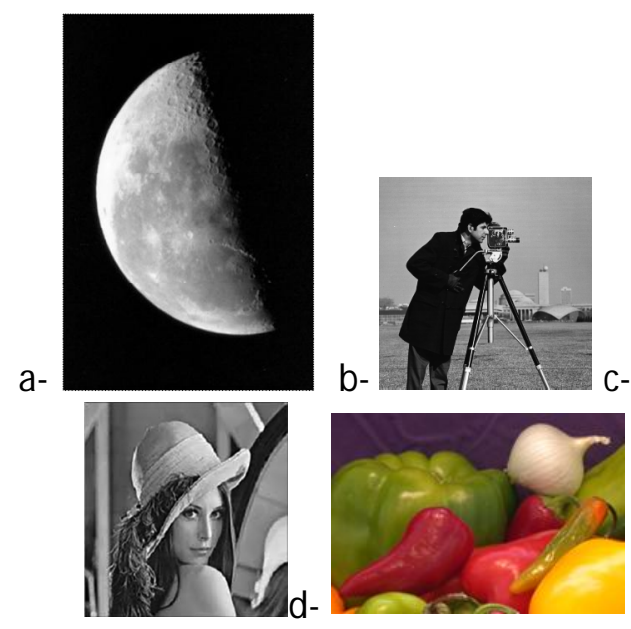

Fig 1:sample of images .
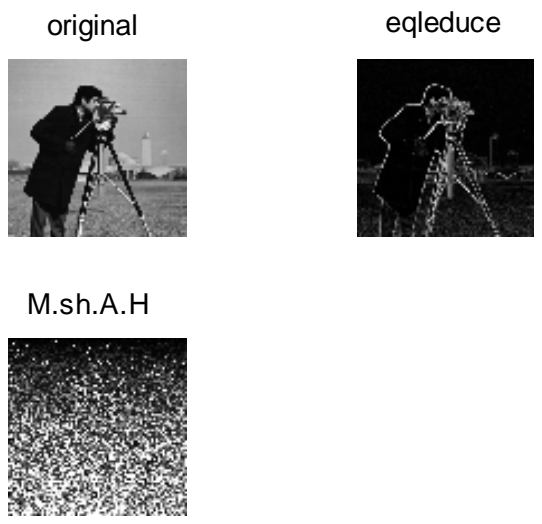

original

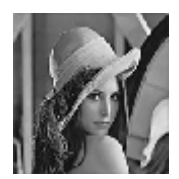

M.sh.A.H

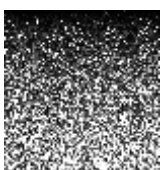

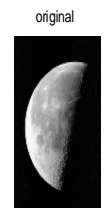

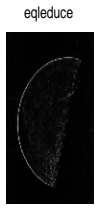

Shahad.A.H
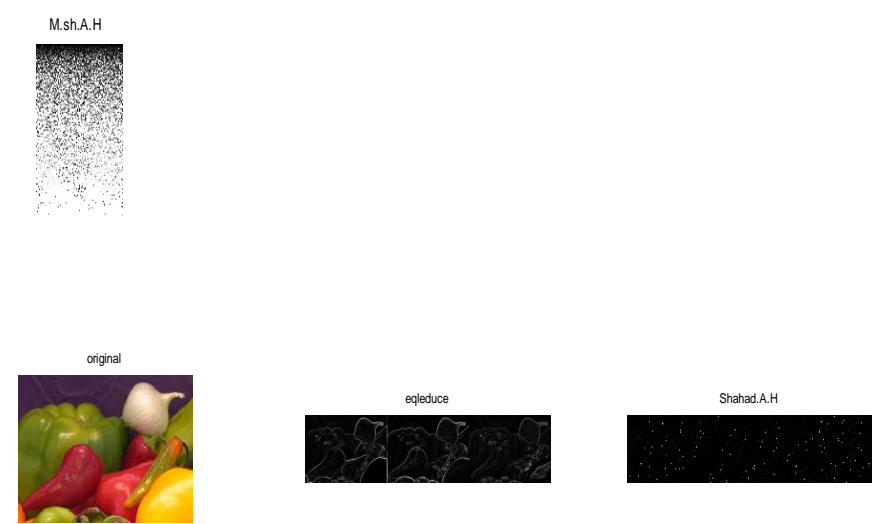

Shahad.A.H

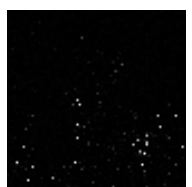

Fig 2: Effect of three methods on the original images.

Figure 2 shows the effect of the methods on the original images. It is shown that the modified method gives the best results and is the most effective in hiding the internal parts of the image.

\section{Histogram:}

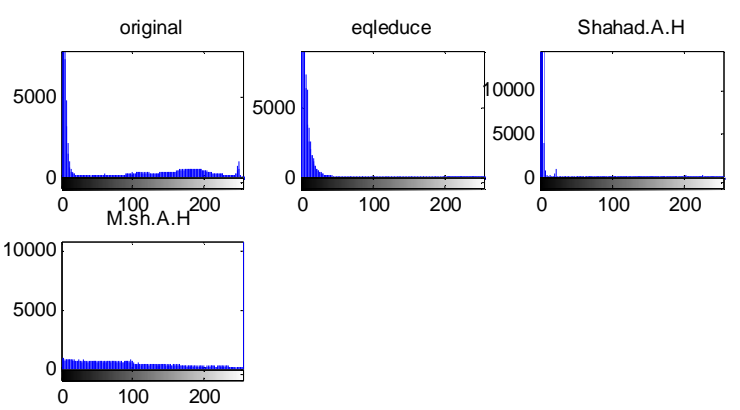



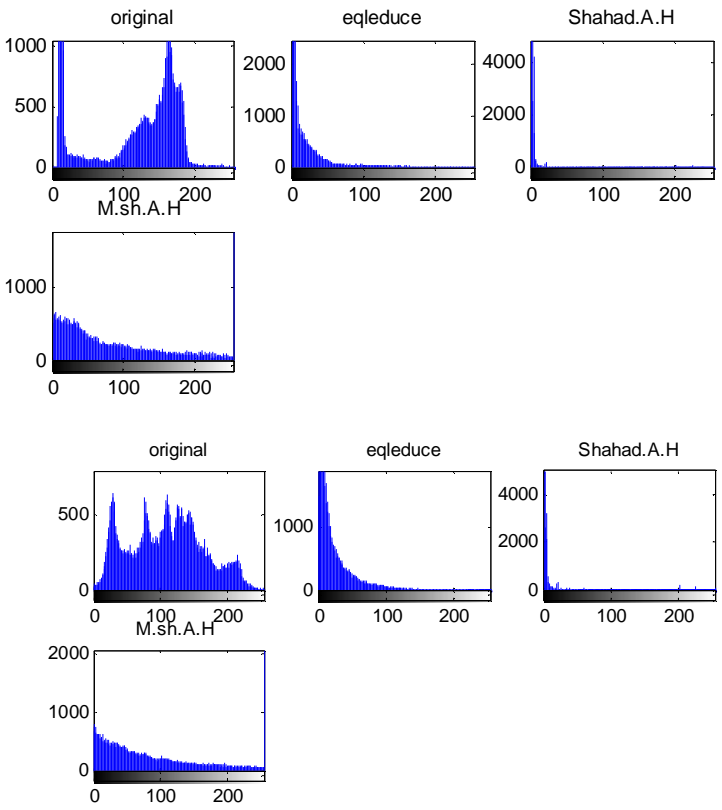

Fig 3 : effecting of histogram on a ,b, c images with 3 methods of encryption .

figure number 3 showed the effect of histogram criterion on original and encrypted images. For information read by the histogram Note that the histogram shows that the proposed method is that which yields a soft results, not as the other methods .

\section{Histogram equalization:}
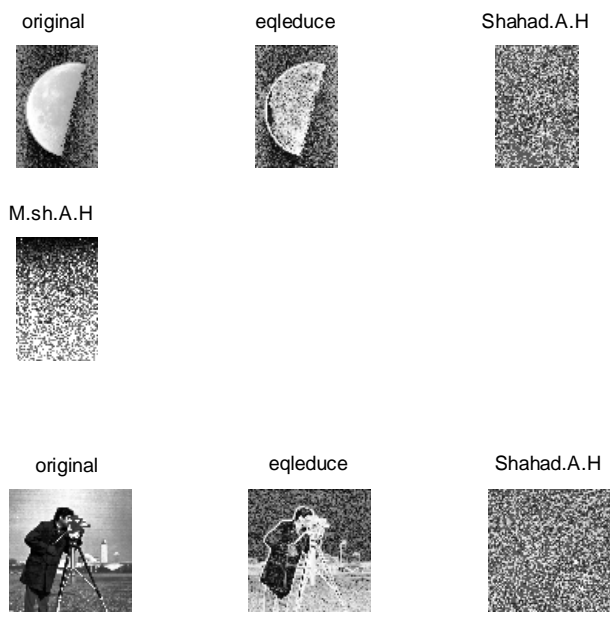

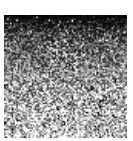

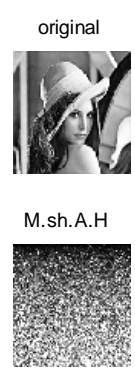

Fig 4 : Effecting of histogram equalization on a ,b, c images with 3 methods of encryption .

Figure 4 exhibits the original image and the encrypted images, shows the difference between the images that had been encrypted by the previously mentioned methods and the image that is encrypted by modify Shahad. A.H .whatever the type of image, or indexed or bilateral, colored or gray, with the

different extension of image. such that the modify method is more active than Shahad.A.H.

\section{Variance (VAR):}
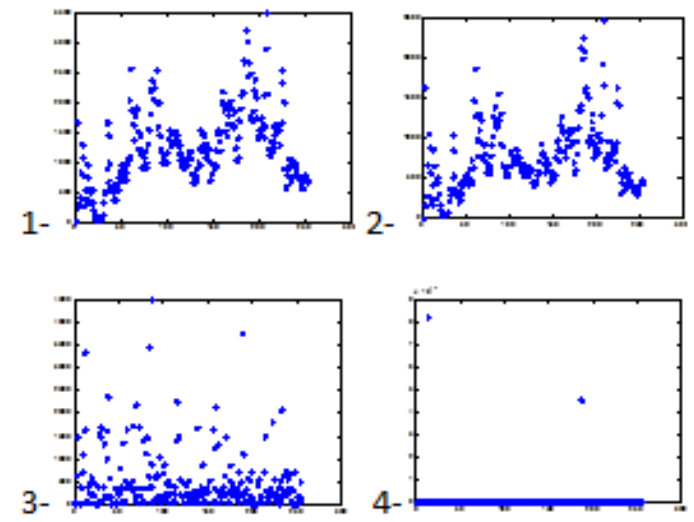

Fig 5 : variance of data on c image such that 1:original image ,2: Euclidean,3: Shahad. A.H,4: M Sh. A. H

Figure 5 The data is distributed in relation to their position in the image and confirms that the proposed method is the best in terms of the spread of data since the data are generated in a uniform and straight way, unlike the previous methods.

\section{ANALYSES OF PERFORMANCE MEASURES}

In this section we calculate the performance of the modification of Shahad. A.H method by using RMSE, (PSNR) ,Correlation Coefficient, and (SSIM) as show in Tables 1,2,3,4. 
Table 1: Showed the performance results of Peak Signal to Noise Ratio( PSNR).

\begin{tabular}{|l|l|l|l|}
\hline $\begin{array}{l}\text { co } \\
\text { d }\end{array}$ & Euclidean & Shahad. A.H & M Sh. A. H \\
\hline a & $\begin{array}{l}34.410723529726 \\
454\end{array}$ & $\begin{array}{l}21.2140999226 \\
29433\end{array}$ & 3.373775936194115 \\
& & & \\
\hline b & $\begin{array}{l}27.606211306855 \\
975\end{array}$ & $\begin{array}{l}14.0544434646 \\
36073\end{array}$ & 6.278636405757673 \\
\hline c & 28.079728229164 & 14.9360313845 & 6.133091285385001 \\
& 278 & 46947 & \\
\hline d & 30.453610330011 & $\begin{array}{l}17.2286851575 \\
08146\end{array}$ & 8.709087234894724 \\
\hline
\end{tabular}

Table 1 appear the results of performance which demonstrated the efficiency of the modify Shahad. A.H method of coding with Peak Signal to Noise Ratio (PSNR) of the three method .

Table 2: showed the performance results of Structural Similarity Index ( SSIM).

\begin{tabular}{|l|l|l|l|}
\hline $\begin{array}{l}\text { co } \\
\text { d }\end{array}$ & Euclidean & Shahad. A.H & M Sh. A. H \\
\hline a & 0.9618885189053 & 0.53924002611 & 0.010713913145305 \\
& 27 & 3141 & \\
\hline b & 0.9554986765149 & 0.36532015936 & 0.021645694690298 \\
& 12 & 1554 & \\
\hline c & 0.9524325179392 & 0.22386114064 & 0.011517031160739 \\
& 55 & 3410 & \\
\hline d & 0.9442483454187 & 0.21979679470 & 0.028977453430396 \\
& 79 & 4248 & \\
\hline
\end{tabular}

While Table 2 explain the results which demonstrated the efficiency of (M Sh. A. H )method of coding with Structural Similarity Index(SSIM).

Table 3 : showed the performance results of correlation coefficients .

\begin{tabular}{|l|l|l|l|}
\hline $\begin{array}{l}\text { co } \\
\text { d }\end{array}$ & Euclidean & Shahad. A.H & M Sh. A. H \\
\hline a & 0.9940202971970 & 0.05580027021 & 0.023943646224944 \\
& 37 & 9064 & \\
\hline b & 0.9915556299465 & 0.04855388794 & 0.012541526903357 \\
& 57 & 5003 & \\
\hline c & 0.9906985563878 & 0.04154368984 & 0.008775525576450 \\
& 09 & 8335 & \\
\hline d & 0.9940202971970 & 0.05580027021 & 0.023943646224944 \\
& 37 & 9064 & \\
\hline
\end{tabular}

Table 3 showed the performance results of correlation coefficients where that appear the modifying method is better than the other methods .

Table 4: showed the performance results of (RMSE).

\begin{tabular}{|l|l|l|}
\hline cod & Shahad. A.H & M Sh. A. H \\
\hline a & $1.277051414210083 \mathrm{e}-11$ & $7.134906404702797 \mathrm{e}-05$ \\
& & \\
\hline b & $1.767054995272250 \mathrm{e}-07$ & $4.270838899999329 \mathrm{e}-05$ \\
& & \\
\hline c & $7.358970315172837 \mathrm{e}-10$ & $8.590290099502474 \mathrm{e}-06$ \\
& & \\
\hline
\end{tabular}

Table 4 showed the performance results of Root Mean Square Errors of the Shahad.A.H method and M Sh. A. H method where the last method had The highest error rate.

\section{CONCLUSION.}

Note that the modified method is the best and most effective for all the criteria used, as well as is easy to use as it depends on finding the inverse of the old method and beating it with the expected value of coordinates,

When looking at tables 1, 2, 3 and 4 note that the modified method gives an effective clear-cut evaluation of performance parameters .

Where the table shows that the lowest value for PSNR is for the proposed method and this links to Table 4 where the relationship is inverse between PSNR and RMSE .

Table 2 illustrates the similarities between the original image and the images under the influence of the above methods Where we note that the lowest amount recorded by the proposed method, as well as Table 3 shows that the weak correlation between the reference image and the image under the influence of the proposed method Compared to other methods s.t less value belong to M SH.A.H method (i.e. good encryption).

\section{REFRENCES}

[1] H. R. Mohammed, I. R. Alsaiq and A.A. Mezher, "Performance of Data Images Encryption Based Karhunen-Leoeve Transforms," Maxwell Scientific Organization Corp. Research Journal of Applied Sciences, Engineering and Technology 14(3): 95-100, 2017.

https://doi.org/10.19026/rjaset.14.4151

[2] R.M. Halagowda1 and S. K. Lakshminarayana, "Image Encryption Method based on Hybrid Fractal-Chaos Algorithm," International Journal of Intelligent Engineering and Systems, Vol.10, No.6, 2017.

[3] Sh.Jaryal and Ch. Marwaha , "Comparative Analysis of Various İmage Encryption Techniques," International Journal of Computational Intelligence Research, SSN 0973-1873 Volume 13, Number 2 (2017), pp. 273-284.

[4] M. M. Deza ,E. Deza , "Encyclopedia of Distances," Springer-Verlag Berlin Heidelberg 2009.

https://doi.org/10.1007/978-3-642-00234-2

[5] R. Zhang, Z. Zhang, K. Liu , J. Zhang, "Proceedings of 3rd International Conference on Logistics", Informatics and Service Science, Liss2003.

[6] Sh. A. Taher, H. R. Mohammed, "Combination Mathematical Distance Measure Approach For Some Image Processing Applications", Journal of Theoretical and Applied Information Technology,30th April 2018. Vol.96. No 8 [7] A. Horé and D. Ziou, "Image quality metrics: PSNR vs. SSIM,", IEEE Computer Society, ISBN 978-0-7695-4109-9,2010.

[8] J. L. Rodgers, W.A. Nicewander, "Thirteen Ways to Look at the Correlation Coefficient," The American Statistician, Vol. 42, No. 1, pp.59-66, February 1988.

https://doi.org/10.2307/2685263

[9] Z. Wang, A.C. Bovik , H.R.Sheikh, and E.P.Simoncelli, "Image quality assessment:From error measurement to structuralsimilarity", IEEE Transaction on Image Processing, 13 (4), 600-612,2004.

https://doi.org/10.1109/TIP.2003.819861

[10] R. E. Walpole, R. H. Myers, Sh .L. Myers

"Probability \& Statistics for Engineers \& Scientists," $9{ }^{\text {th }}$ EDITION, ISBN 10: 0-321-62911-6 ISBN 13: 978-0-321-62911-1

[11] T. Chai1,2 and R. R. Draxler1, "Root mean square error (RMSE) or mean absolute error (MAE) - Arguments against avoiding RMSE in the literature," Published by Copernicus Publications on behalf of the European Geosciences Union. 30 June 2014.

[12] M. L. Najman, J. Facon, and A. de A. Ara ujo,

"Multi-histogram equalization methods for contrast enhancement and brightness preserving," Consumer Electronics, IEEE Transactions on, vol. 53, no. 3, pp. 1186-1194,2007. 
SHAHAD ADIL TAHER et al., International Journal of Information Systems and Computer Sciences, 7(5), September - October 2018, 19- 24

[13] A. A. Rani1 ; G. Rajagopal2 ; A.Jagadeeswaran3 , "Bi-Histogram Equalization with Brightness Preservation Using Contras Enhancement", International Journal of Basic and Applied Science, Vol. 02, No. 03, ,pp.21-

30 Jan 2014.

[14] H.S. Kwok, Wallace K.S. Tang "A fast image encryption system based on chaotic maps with finite Precision representation,". Chaos Solitons Fractals 32(4), 1518-1529 (2007)

https://doi.org/10.1016/j.chaos.2005.11.090 